\section{Cost of the charge}

SEQUESTERING a charged side-chain in the low-dielectric-constant heart of a globular protein has always been surmised to exact a ruinous thermodynamic cost. Sometimes paired charges have evolved to reduce this penalty. Now S. Dao-pin et al. (Biochemistry 30, 11521-11529; 1991) have made two mutants of T4 phage lysozyme, one with lysine for methionine on the inward-facing surface of an $\alpha$ helix, the other with aspartate for a leucine. The conformations are less stable, but both chains fold, giving products with 35 per cent and 4 per cent activity. The first mutant has been crystallized and the violated helix is appreciably wobbly. The pK of the intruding lysine is 6.5 and the structure survives down to $\mathrm{pH} 3$. Such mutations, then, are evidently not as calamitous as has been supposed.

\section{Old story}

CONVENTIONAL thinking has it that clonally reproducing organisms don't last for long in evolutionary terms, at least compared with the sexually reproducing stock from which they stem. Ain't necessarily so, find J. M. Quattro and colleagues in their estimation of the time over which a unisexual, all-female lineage of fish has persisted

(Proceedings of the National Academy of Sciences 89, 348-352; 1992). Quattro et al. looked at 'post-formational' (that is, post-hybridization) mutations in the mitochondrial DNA and allozymes of the unisexual, live-bearing fish Poeciliopsis monacha-occidentalis, which occurs in arroyos (streams) in Mexico where the distributions of its two sexual progenitors overlap. As evidenced by mitochondrial DNA restriction-site data, the lineage manifests a high level of genetic diversity and is, the authors estimate, at least 100,000 generations old.

\section{Steps in time}

L. R. BRAND, together with Thu Tang, has carried out a further appraisal of how the fossil tetrapod trackways in the

Coconino sandstone of northern Arizona came to be formed (Geology 19, 1201$1204 ; 1991)$. At issue is whether the sandstone, which is of Permian age, was deposited subaerially, as sand dunes, or under water. Brand and Tang produced diagrams of the orientation of the limb prints and direction of the fossil tracks, and compared them with those made by newts on sand in a tank of flowing water $4 \mathrm{~cm}$ deep. In many of the fossil tracks, limb impression and track direction are at different angles, a feature also evident in the impressions left by the living newts as they were drifted by the current. That, say the authors, points towards an underwater origin for at least some of the sandstone. bated microsomes with protease. This treatment, the authors argue, should have cleaved the cytoplasmically exposed ATP-binding domain of the postulated transporters. Because no effect on peptide binding to MHC class I molecules was evident, they concluded that transporters are not involved in this process. But because the protease sensitivity of the putative transporters is not known, the results are suggestive rather than conclusive.

The idea that ATP-driven transporters are involved in the transport of peptides across the ER membrane arose from analysis of mutant cells defective in surface expression of class I molecules. Mutant T2 cells derived from the B lymphoblastoid cell line T1 have a deletion in the MHC, and they express class I molecules intracellularly but not on the cell surface. Cerundolo et al..$^{5}$ found that these cells had lost the ability to present intracellular antigen efficiently, whereas they could present extracellular antigen of eight and twelve amino-acid residues efficiently. One possible explanation for the defect in these cells is that peptides derived from cytosolic proteins do not reach the lumen of the ER. To test this possibility, Lévy et al. investigated the ability of microsomes from T1 and T2 cells to take up peptides and assemble them with class I molecules. They found that peptides were taken up into microsomes from $\mathrm{T} 1$ and $\mathrm{T} 2$ cells with similar efficiencies. But peptide-stimulated assembly of class I molecules was only seen with microsomes of T1 cells, not with those of mutant $\mathrm{T} 2$ cells. Lévy et al. propose that the defect is in luminal factors needed for peptide binding to the class I molecules.

The authors consider several explanations for the defect in the lumen of microsomes from $\mathrm{T} 2$ cells and the ATP requirement for peptide binding to class I molecules. Peptides may become concentrated by binding to $\mathrm{BiP}$ and the ATP is needed to release them (see figure). I consider this to be unlikely, because peptide would re-enter the free-peptide pool after release from $\mathrm{BiP}$. Peptides may require trimming in the lumen of the ER before binding to class I molecules. This possibility remains open, however, and needs further experiments with peptides of different size.

Another possibility is that the oligomeric assembly of MHC class I molecules needs a catalyst for proper folding. This is the most likely explanation for an ATP-dependent step, as experimental evidence for a role of ATP for folding and disulphide-bond formation in proteins in the ER will shortly be described by Braakman and colleagues $^{12}$. These authors demonstrate that folding of a viral glycoprotein in the ER is an energy-dependent process. In the absence of ATP proteins misfold into disulphide cross-linked aggregates, but they can be rescued and fold correctly when cells are allowed to regenerate ATP again. Class I heavy chains and $\beta_{2}$-microglobulin may also require ATP for their folding and oligomerization, and may aggregate in the absence of ATP. Under such conditions peptide binding would not occur.

Is there really no requirement for peptide transporters? Indeed for peptides up to about ten amino acids in length there may be no requirement for active transport across microsomal membranes. But a difference in the permeability of small peptides across membranes of isolated microsomal vesicles from that of the ER membrane in intact cells cannot be ruled out. It will be interesting to see whether peptides applied to the cytosol of cells by either micro-injection or after permeabilization of the plasma membrane can cross the ER membrane in an ATP-independent manner.

The results of Lévy et al. raise another important question. What size are the peptides present in the cytosol? In particular, are they the right size to interact with MHC class I molecules? Little is known about the peptide intermediates in the degradation of cytosolic proteins. It is conceivable that proteasomes proteolytically process cytosolic proteins to fragments which can be actively transported across the ER membrane. To draw a more definite conclusion about the requirement for peptide transporters, intact protein substrates, not just trimmed peptides, should be investigated for their requirements for proteolytic processing and membrane translocation. With the in vitro system used by Lévy et $a l$., it should be possible to analyse the entire pathway of proteolytic processing of cytosolic proteins, transport of peptides across the ER membrane and binding to the MHC class I molecules. It is likely that several energy-requiring steps and factors mediating charging of MHC class I molecules with peptides still await discovery.

Bernhard Dobberstein is in the European Molecular Biology Laboratory, Meyerhofstrasse 1, 6900 Heidelberg, Germany.

Robertson, M. Nature 353, 300-301 (1991)

2. Parham, P. Nature 348, 674-675 (1990).

3. Lévy, F., Gabathuler, R., Larsson, R. \& Kvist, S. Cell 67, 265-274 (1991).

4. Neefjes, J. J.. Schumacher, N. M. \& Ploegh, H. L. Current Biol. 3, 601-609 (1991).

5. Cerundolo, V. et al. Nature 345, 449452 (1990)

6. Spies, T. \& DeMars, R. Nature 351, 323-324 (1991).

7. Gilmore, R. Current Biol. 3, 580-584 (1991).

8. Wiech, H., Stuart, R. \& Zimmermann, R. Seminars Cell Biol. 1, 55-63 (1990).

9. Lau, J. T. Y. et al. J. biol. chem. 258, 15255-15260 (1983).

10. Lévy, F., Larsson, R. \& Kvist, S. J. Cell Biol. 115 959-970 (1991).

11. Pelham, R. B. A. Rev. Cell Biol. 5, 1-23 (1989).

12. Braakman, I., Helenius, J. \& Helenius, A. Nature (in the press).

NATURE $\cdot$ VOL $355 \cdot 9$ JANUARY 1992 